Revue d'histoire du XIXe siècle

Société d'histoire de la révolution de 1848 et des

révolutions du XIXe siècle

$22 \mid 2001$

Autour de Décembre 1851

\title{
LA PROSCRIPTION ET LES ORIGINES DE 'INTERNATIONALE II
}

Les alliances des Blanquistes dans la proscription

Norman Plotkin

\section{(2) OpenEdition}

Journals

Édition électronique

URL : http://journals.openedition.org/rh19/257

DOI : 10.4000/rh19.257

ISSN : $1777-5329$

Éditeur

La Société de 1848

Édition imprimée

Date de publication : 1 juin 2001

ISSN : 1265-1354

Référence électronique

Norman Plotkin, "LA PROSCRIPTION ET LES ORIGINES DE 'INTERNATIONALE II », Revue d'histoire du XIXe siècle [En ligne], 22 | 2001, mis en ligne le 27 juin 2005, consulté le 02 mai 2019. URL : http:// journals.openedition.org/rh19/257; DOI : 10.4000/rh19.257

Ce document a été généré automatiquement le 2 mai 2019.

Tous droits réservés 


\title{
LA PROSCRIPTION ET LES ORIGINES DE 'INTERNATIONALE II
}

\author{
Les alliances des Blanquistes dans la proscription
}

\author{
Norman Plotkin
}

1 Pendant la longue période d'emprisonnement que subit Blanqui après la Révolution de 1848, l'organisation qu'il dirigeait en France fut effectivement brisée et ses membres dispersés. La plus grande partie de ses partisans qui échappèrent à l'arrestation se réfugièrent en Angleterre avec les autres proscrits de 1848. Une activité politique intense entraîna les exilés français de tous les partis. Mais Londres était en même temps le centre des révolutionnaires émigrés de toute l'Europe, et les relations internationales formées en France pouvaient se renouveler et s'élargir.

2 Ce fut aussi une époque de grande agitation dans la vie politique anglaise : le mouvement chartiste, en se désintégrant, laissa d'importantes forces révolutionnaires qui cherchaient à s'allier aux mouvements du continent. De nombreux comités et sociétés furent créés rapidement; mais ils ne survécurent pas à leurs différences d'idéologies, bien que leur existence passagère préparât le chemin pour d'autres organisations plus durables.

3 Les Blanquistes qui se réfugièrent à Londres se mêlèrent à ces activités tant dans les milieux français que dans les groupements étrangers. Des rapports sur ces relations furent communiqués à Blanqui, qui se tint ainsi au courant des activités de ses collègues. On peut lire donc, dans les lettres à Blanqui de cette époque, bien des détails de la vie des émigrés à Londres. Et dans ses réponses, Blanqui donne des conseils et parfois des admonestations, comme dans l'affaire de l'“ Avis au Peuple " du 1851.

4 Dès le début de 1850, son ami Flotte vit plus clair que d'autres révolutionnaires et comprit que les divers éléments unis par leur exil commun ne pouvaient pas constituer un parti solide; en cela, il est évident que Flotte partagea les opinions de Blanqui, bien qu'il l'ait peu connu à cette époque. Écrivant ses impressions générales sur le milieu des réfugiés dans une lettre à Blanqui de février 1850, il prévit la rupture qui devait éclater au sein de leur organisation unie. Mais c'est aussi avec un esprit optimiste qui devait réjouir Blanqui, isolé dans sa cellule à Doullens, que Flotte écrivit : “... Il est encore une autre 
consolation [...] je suis heureux de pouvoir vous la donner : c'est celle qui naît des progrès réels accomplis par notre cause. Tout marche, cher, et tout marche bien, les idées et les hommes. Il se produit dans la société entière un grand mouvement. D'une part l'unité se fait peu à peu dans les doctrines parmi les hommes de pensée qui, quoi qu'il arrive, seront nécessairement, tôt ou tard, les directeurs de l'action révolutionnaire ".

“Entre temps, la propagande fait des merveilles, et le nombre des démocrates va toujours grandissant. Mais il ne faut pas perdre de vue que cette double action agit dans des milieux parfaitement distincts. La propagande est multiple, s'adresse à tous les esprits, prend toutes les formes, et nous assistons à ce singulier spectacle de voir toutes les formules révolutionnaires qui se sont succédées, servir au recrutement d'une opposition qui se croit unie ; qui en apparence n'a qu'un drapeau et dans laquelle on peut trouver actuellement unies (si l'on passe des hommes les plus intelligents à ceux qui le sont le moins) toutes les nuances de l'esprit de la révolution, depuis celui de l'avenir jusqu'à celui du passé. C'est là une situation difficile, et qui nous prépare pour l'avenir bien des luttes non seulement au dehors, mais dans le sein du parti même" ${ }^{1}$.

6 À cette juste analyse, Flotte ajouta un autre jugement historique qui fut non moins vrai. Il conseillait, de façon certaine et optimiste, l'abandon des vieilles méthodes de conspiration: "Pas de sociétés secrètes maintenant, pas de conciliabules. Nous allons gouverner. Il faut perdre les habitudes de la lutte et prendre celles du calme et de la force ". Car, en effet, depuis la révolution de 1848, les Blanquistes n'étaient plus un petit parti isolé ; les chefs mêmes de l'ancien gouvernement provisoire s'unirent avec eux dans leur exil commun.

7 La forme concrète de cette unité fut, à ce moment-là, une société dite démocratesocialiste, qui groupa tous les proscrits français. Dans la même lettre, Flotte communiqua à Blanqui “... la composition que vous devez connaître de la liste du comité démocratesocialiste : moi, Vidil, Carnot".

8 L'opinion de Flotte sur les luttes à venir fut confirmée plus tard dans la même année par Barthélémy ; mais ce dernier, cependant, ne présenta pas un tableau si encourageant. Une lettre de Blanqui à Goûté, datée du 29 juin, fut communiquée à Barthélémy. Celui-ci répondit le 4 juillet $1850^{2}$, se plaignant de l'influence de Louis Blanc et de Caussidière, qui, “à leur arrivée à Londres, trouvèrent de la sympathie parmi les Anglais chartistes ou disciples de Robert Owen", et racontant la scission qui s'était produite dans leur organisation unie. Cazavant et Dupont "formèrent, après avoir consulté L. Blanc et Caussidière, le dessein de fonder une société dont le but serait de protéger les réfugiés [...] Tant qu'il ne s'était agi que de questions de secours, tout le monde avait été d'accord. Mais les discussions s'étaient étendues jusqu'aux principes, une scission se fit, et nous fûmes aussitôt partagés en deux camps : celui des riches fondateurs et celui des pauvres affiliés". "Si les sceptiques, déplorait-il, avaient écouté les prophéties de la Société Républicaine Centrale!"

9 Après la scission, poursuit la lettre, une autre organisation fut créée, et une lutte eut lieu entre les deux groupes. "Dans le même temps, ajouta Barthélémy, je faisais partie avec Adam d'une autre société composée d'ouvriers seulement, et qui s'était formée à Londres après la révolution de février. Nous eûmes l'idée de faire entrer tous les ouvriers de cette société dans celle des proscrits". Cette proposition devait soulever l'opposition au sein même de cette organisation, où "Maillard, qui fit, dans le temps, partie de la Société des Saisons [...] était surtout enragé parce que, disait-il, je soufflais l'âme de Blanqui dans la société". 
10 L'absence des membres riches dans la nouvelle organisation des proscrits souleva de sérieuses difficultés financières et Barthélémy évoqua les souffrances matérielles des réfugiés ; celles-ci cependant, n'étaient pas leurs uniques soucis. “Mais, citoyen, écrivit-il, je crois que c'est assez sur nos misères physiques. Quelque chose de plus désolant que tout cela, c'est le peu de foi de la plupart des proscrits de l'émigration. Les idées de la Société centrale sont peu estimées ici ; nous n'avons jamais été plus de 6 ou 7 amis de son ancien président. C'étaient Louis Menard (du Peuple), Larger, Watripon et un ou deux autres qui vous sont inconnus. Maintenant ce sont: Vidil (capitaine du $6^{\mathrm{e}}$ Hussards), Cottier (l'ancien vice-président du Club des Montagnards de Belleville), Goûté et moi [...] "

11 Blanqui fut certainement inquiété par cette communication si décourageante, car il chercha à vérifier les nouvelles transmises par Barthélémy. Quelques semaines plus tard, une réponse lui parvint de Vidil. Ce dernier, dans sa lettre à Blanqui du 19 juillet $1850^{3}$, confirma la scission et l'hostilité entre les deux camps; mais son esprit, loin d'être pessimiste comme celui de Barthélémy, est tout autre.

12 "Je vais essayer de vous distraire, écrivit-il, dans votre tombeau vivant, en complétant, autant que je pourrai, les renseignements de B. sur la situation de l'émigration à Londres. G. vous est très dévoué, j'en suis convaincu, mais il est très paresseux; aussi m'a-t-il chargé de compléter sa lettre par la plus longue épître possible sur ce qui se passe ici.

13 "Vous demandez si nous sommes nombreux dans notre nuance et vous supposez que la presque totalité des réfugiés suit la bannière de l'ancien gouvernement provisoire. Il n'en est heureusement rien. Ce qui est la Société des Proscrits Démocrates-socialistes est essentiellement communiste. La fraction la plus énergique, sans contredit, se dit et est de fait blanquiste. Deux membres en font partie: Watripon et Menard. Il est bien entendu qu'en disant blanquistes on spécifie vos idées révolutionnaires et votre conduite depuis le 24 février.

14 “Voilà, je crois, l'idée exacte de notre société, de laquelle a dû nécessairement s'éloigner toute l'aristocratie du parti. L. Blanc et Landolphe, aussi communistes, se sont retirés d'avec nous, pour les faits d'amour-propre et des questions personnelles, disent-ils. Mais il est à craindre qu'il n'en soit autrement, et qu'ils n'aient voulu ne pas faire scission complète avec les grands princes de la démocratie, et avec la Montagne de Paris, de laquelle le dernier reçoit un subside, comme ancien représentant".

15 D'autres scissionnistes furent cités dans cette lettre: Ledru-Rollin, Martin-Bernard, Rattin "que vous ne connaissez pas [...] un vrai propre à rien qui oublie son origine comme travailleur" et Delescluze "qui vient, dans leur journal mensuel, intitulé Le Proscrit, d'insulter le prolétariat et le socialisme de la manière la plus dégoûtante". Continuant la liste, Vidil écrit: "Il y a aussi ici E. Arago, Heitzman, représentant de Saône-et-Loire, ouvrier mécanicien, seul de son parti. Je n'en dis rien, vous en savez assez. Il est possible que j'en oublie. Tous ces hommes se trouvaient parmi nous et nous ont abandonnés".

16 Pour compléter le tableau, il indique l'existence d'un troisième parti. "Il y a bien encore un petit parti, placé entre nous et la Montagne. Ce parti est communiste, mais toujours bourgeois, et s'ils ne veulent plus de privilèges pour les autres, il est à craindre qu'ils n'en veulent pour eux. Madier de Montjeau jeune, Langlois, Pardigon, Berjeau, voilà de quoi se compose ce parti".

17 Par cette série de lettres on peut suivre, aussi bien que Blanqui dans sa prison à Doullens, les relations entre son parti et les "démocrates" de cette époque. On comprend ainsi 
l'évolution de la pensée de Blanqui qui aboutit à son "Appel au Peuple" de janvier 1851. Dans son toast de décembre 1848 il se déclarait "socialiste" et proclamait que "la Montagne est morte"; par la suite, vues les activités des proscrits et la situation en France, il s'inquiéta de ce " cadavre " qu'auparavant il se contentait d'enterrer ; après les confusions et les hésitations de ses amis à Londres il comprit que la Montagne était encore à tuer. D'ailleurs, Blanqui ne fut ni le seul, ni le premier à se rendre compte de cet antagonisme aigu, si évident à Londres que Vidil terminait sa lettre du 19 août ainsi : "Nous sommes pour eux (L. Blanc et al.) des Hébertistes; eux, disent-ils, sont des Montagnards-Jacobins".

18 À cette même époque, les blanquistes de Londres participèrent à la formation d'une organisation internationale avec des communistes allemands. Ce n'était pas la première fois qu'ils avaient eu de telles relations ; en 1847, le blanquiste Jacques Imbert avait pris part à une organisation internationale démocratique à Londres ${ }^{4}$, et déjà en France, selon Nicolaievsky, " dans les groupes blanquistes il existait même des sections allemandes " ${ }^{5}$; ce fut une période, on se souvient, où un grand nombre de travailleurs allemands étaient venus comme immigrés. Karl Marx, pendant son séjour à Paris en 1844 et 1848 fréquenta ce milieu où il fut introduit par le cabétiste Everbeck ${ }^{6}$ le dirigeant "des groupes allemands de Paris" ?.

19 En Allemagne aussi, les doctrines blanquistes inspiraient certaines organisations révolutionnaires. Charles Andler démontre l'identité de forme et de programme de la Fédération des Bannis d'Allemagne avec la Société des Saisons en France; il conclut que les clubs de Blanqui étaient pris comme modèles de société secrète ${ }^{8}$. Cette influence fut particulièrement importante, affirme Nicolaïevsky ; il est bien possible, écrit-il, que celuici “ était en relations avec Blanqui ; selon le témoignage de Herweg, lorsque Gottschalk se rendit à Paris au début de 1849, il alla voir Blanqui dans sa prison " 9 .

20 Cependant, jusqu'en 1850, les blanquistes n'avaient jamais été formellement alliés aux organisations étrangères. Mais en cette année, la Société Universelle des Communistes Révolutionnaires fut créée à Londres; elle comprenait des blanquistes, des marxistes et des chartistes. Son importance fut considérable, malgré sa courte existence ; d'une part, elle acheva l'unité des partis européens de gauche après la révolution de 1848 ; d'autre part, elle marqua le premier pas vers une organisation internationale révolutionnaire durable.

21 Blanqui fut informé de cette alliance par Barthélémy, dans sa lettre du 4 juillet 1850, citée ci-dessus : "Nous avons commencé conjointement avec les Communistes Allemands, la rédaction d'un manuel révolutionnaire contenant par ordre de numéros, toutes lés mesures que le peuple devra prendre immédiatement après la révolution [...] Notre intention est de faire de ce manuel un petit livre que nous répandrons parmi les ouvriers [...] Nous ferons aussi imprimer ce manuel en forme de proclamations pour l'afficher dans les rues de Paris. Faites-nous savoir si nous pouvons vous faire passer notre manuscrit sans inconvénient pour que vous puissiez y mettre la dernière main. En le lisant vous verrez quelles sont nos intentions, et je ne doute pas, pour mon compte, que vous ne les approuviez [...] Écrivez-nous pour le manuel en question ".

Vidil aussi dans sa lettre du 19 juillet, transmit à Blanqui le même renseignement: "Barthélémy vous promet de vous soumettre un petit travail que nous avons fait ici; vous le recevrez par la première occasion. Ce travail contient ce que le peuple doit exiger le lendemain d'une révolution. Le jour même de la victoire, il sera affiché dans tout Paris. Mais d'ici là notre intention est d'en faire imprimer des façons de petits manuels que nous 
ferons répandre gratis dans tous les ateliers de Paris. L'impression sera faite ici. Ce livre déplaira à nos Montagnards qui nous appellent des hommes de désordre..."

Ce manuel n'a pu encore être retrouvé ; on ne sait même si Blanqui le reçut. Mais on connaît le texte, rédigé en français, des statuts de la Société Universelle des Communistes Révolutionnaires ${ }^{10}$. Ils comprennent six articles :

24 ARTICLE PREmier. - " Le but de l'association est la déchéance de toutes les classes privilégiées, de soumettre ces classes à la dictature du prolétariat en maintenant la révolution en permanence jusqu'à la réalisation du communisme, qui doit être la dernière forme de constitution de la famille humaine".

25 ART. 2. - "Pour contribuer à la réalisation de ce but, l'association formera des liens de solidarité entre toutes les fractions du parti communiste révolutionnaire en faisant disparaître conformément au principe de la fraternité les divisions de nationalité ".

26 ART. 3. - "Le Comité fondateur de l'association est constitué en comité central; il établira partout où besoin sera, pour l'accomplissement de l'œuvre, des comités qui correspondront avec le comité central ".

27 ART. 4. - "Le nombre des membres de l'association est illimité, mais aucun. membre ne pourra être admis, s'il n'a pas réuni l'unanimité des suffrages. Dans aucun cas l'élection ne pourra avoir lieu au scrutin secret".

28 ART. 5. - “Tous les membres de l'association s'engagent par serment de maintenir dans les termes absolus l'article premier du présent règlement. Une modification pouvant avoir pour conséquence l'affaiblissement des intentions exprimées dans l'article premier délie les membres de l'association de leur engagement.

29 ART. 6. - "Toutes les décisions de la société sont prises à la majorité de deux tiers des votants" 11 .

30 On voit, d'après les principes et l'organisation prévue dans les statuts, jusqu'à quel point étaient identiques les points de vue des trois partis constituants. Le programme reste essentiellement politique, car aucune mesure économique pour réaliser "le communisme " n'y est précisée. Quant à la forme d'organisation, on remarque l'absence des conceptions particulières aux blanquistes, ou aux autres, qui auraient rendu impossible une telle alliance. Probablement, elle n'eut d'autre but que de faire de la propagande, et d'une façon générale, de préparer le peuple idéologiquement pour la prochaine révolution. Cependant, d'après Nicolaïevsky, la Société Universelle était, en quelque sorte, un centre insurrectionnel pratique. "Les Fraternal Democrats, écrit-il, pouvaient être utiles pour établir des liaisons entre différents cercles d'études, mais ils étaient inaptes à la nouvelle tâche. Il fallait créer une union de sociétés secrètes en vue de l'action commune lors de la révolution qui pouvait éclater d'un instant à l'autre [...] En avril 1850, naquit l'organisation internationale de combat [...] la Société Universelle des Communistes Révolutionnaires".

31 Blanqui, depuis longtemps, avait abandonné la méthode de société secrète; et il n'est guère concevable, que dans cette période de victoire générale de la contre-révolution, les blanquistes aient projeté une tentative immédiate d'insurrection. Marx n'était certainement pas partisan de cette conception; Nicolaïevsky lui-même le démontre : au moment où Marx "signa l'accord avec les blanquistes [...] parut le numéro de la Neue Rhenische Zeitung, sous forme de revue [...] (qui) contenait la critique des livres de A. Chenu et de Lucien de la Hodde. Marx y jugeait impitoyablement les conspirateurs 
professionnels" ${ }^{12}$. L'affirmation que la Société Universelle est une société secrète est un véritable non-sens.

32 Si la raison de sa dissolution n'est pas certaine, on sait qu'à cette époque les Communistes allemands se sont disputés sérieusement entre eux, et ont suspendu leur propre organisation, cause plus probable de liquidation que les désaccords entre Blanquistes et non-Blanquistes.

33 Cette société est importante par l'alliance des blanquistes et des marxistes qu'elle montra, plutôt que par la quantité de ses réalisations, bien qu'il y ait à son compte, au moins, "la rédaction d'un manuel révolutionnaire " et sa diffusion à Paris, signalées dans les lettres de Barthélémy et de Vidil.

\section{NOTES}

1. P. B. $9583-9584^{1}$ fo $^{\circ} 133$ (verso) ; $9584^{2}$ f $^{\circ} 401$.

2. P. B. $9.582 \mathrm{f}^{\circ} 5 ; 9581-82 \mathrm{f}^{\circ} 200 ; 9584^{2} \mathrm{f}^{\circ} 371$.

3. P. B. $9582 \mathrm{f}^{\circ} 8$; $9583-9584^{1} \mathrm{f}^{\circ} 143$ (verso).

4. NICOLAÏEVSKI et Otto MEIENCHEN-HELFEN, Karl Marx, Paris, 1937, p. 114.

5. Ibid. p. 70.

6. Ibid. p. 73.

7. Charles ANDLER, Introduction historique. Le Manifeste Communiste, Paris, 1901, p. 33.

8. Charles ANDLER, Introduction historique, le Manifeste Communiste. (Papiers Blanqui mis à la Bibliothèque nationale).

9. NICOLAÏEVSKI et MEIENCHEN-HELFEN, Ibid., p. 137.

10. Le texte est reproduit dans les Cahiers du Bolchevisme, mars 1933, p. 405. et par NICOLAÏEVSKY, ibid., p. 175.

11. Document signé de G. Vidil, Aug. Willich, G. Harney, Adam, Ch. Marx et F. Engels.

12. NICOLAÏEVSKI, Ibid., pp. 175 et 178. 